\title{
Morphological and morphometric study of the cartilaginous framework of the dorsum and tip of the nose among Egyptian populations: a cadaveric study
}

\author{
E.A.A. El-Shaarawy \\ Department of Anatomy and Embryology, Faculty of Medicine, Cairo University, Cairo, Egypt
}

[Received: 9 December 2015; Accepted: 28 January 2016]

\begin{abstract}
Background: Success or failure of rhinoplasty depends mainly on the awareness of the surgeon with the construction of the nasal cartilaginous framework and the knowledge of the morphology and different dimensions of these cartilages. The current study aimed at evaluation of the morphology of the nasal cartilages and to address their different measurements as well as observation of anatomical variations of these cartilages and elucidation of their implications in rhinoplasty. Materials and methods: Thirty adult human cadaveric noses, males and females aged 20-70 years were used in the current study. The specimens were dissected; cartilages were exposed and examined morphologically for shape, parts and attachments. Different nasal cartilage measurements were done.

Results: The examination of nasal cartilage revealed that the mean length of upper lateral cartilage was variable. The mean width and thickness were $12.8 \pm 1.29 \mathrm{~mm}$ and $1.34 \pm 0.14 \mathrm{~mm}$, respectively. The mean length, width and thickness of alar cartilage were assessed. Angle of divergence was observed to be $23-44^{\circ}$. Statistically, there were no significant differences between genders.

Conclusions: Data obtained from this study confirmed the anatomical variations of the nasal cartilages among the Egyptians and confirmed the differences with several ethnic groups. This has important surgical implications giving the attention required during different techniques in rhinoplasty in order to maintain dorsal aesthetic lines of the nose and proper respiratory function. (Folia Morphol 2016; 75, 3: 316-325)
\end{abstract}

Key words: upper lateral nasal cartilage, lower lateral nasal cartilage, nasal cartilages' measurements, domal angle

\section{INTRODUCTION}

Rhinoplasty is a precise surgery; therefore the operating surgeon must have a thorough knowledge of nasal anatomy and its inherent variations [3]. The nose like any other projecting structure is made of up three components; framework, support, and external cover [11]. The nasal framework consists of nasal bones, upper and lower lateral cartilages and indirectly the septum while the support is provided by connective tissue and ligaments that hold the intricate framework together. The skin and soft tissue provide the external covering of the nose. These components are intimately related and must be anatomically visualised in every step of the rhinoplasty sequence [1]. de Pochat et al. [13] mentioned that the upper lateral cartilage (ULC) manipulation is often associated

Address for correspondence: Dr E.A.A. El-Shaarawy, Assistant Professor of Anatomy and Embryology, Department of Anatomy and Embryology, Faculty of Medicine, Cairo University, Cairo, Egypt, tel: 01001582811, e-mail: ehab.elshaarawy@yahoo.com 
with compromise of the middle-third vault. They also added that although the anatomical details of the ULCS are of great importance for the maintenance or even the creation of an aesthetically pleasing dorsum with proper respiratory function, the literature includes few studies related to these themes. Crag et al. [7] observed that the ULC articulates laterally with the frontal process of the maxilla by means of the pyriform ligament with a variable amount of overlap between the ULC and maxilla. Rohrich and Ghavami [23] on their study on Middle Eastern noses found that the lower lateral, middle and medial crural cartilages are often weak and thin relative to the heavy skin and soft tissue sleeve. They noted that the lateral crura were commonly wide, thin and that the middle and medial crura were insufficient.

Rowe-Jones [24] described that the contour, position and orientation of the caudal margins of the middle and lateral crura of lower lateral cartilages are a key component in achieving natural tip refinement. Chin and Uppal [4] stressed the fact that preoperative anatomical measurement of the nasal cartilages allows reliable assessment of nasal characteristics and comparison with postoperative outcomes. Farahvash et al. [15] mentioned that significant gender differences were observed for the middle and medial crura but not the lateral crura. They observed that $87 \%$ of lateral crura of alar cartilages were convex, $86 \%$ of middle crura were flat while the medial crus was convex in $58.3 \%$ of the cadavers they examined. They noted occasional asymmetry between the middle and medial crura. They concluded that alar cartilage anatomy varies across ethnic groups and attention to the differences is recommended during preoperative planning and intraoperative technique when treating the nasal tip.

This study aimed to evaluate the morphology of the nasal cartilages and their extension and to address their different dimensions among Egyptian population as well as to examine the anatomical variations of these cartilages and their implications in rhinoplasty.

\section{MATERIALS AND METHODS}

Thirty adult human noses of both sexes (17 male and 13 female, 20-70 years of age) were obtained from the cadavers and used in the current study. The specimens were obtained from the Department of Anatomy and Embryology, Faculty of Medicine, Cairo University and subjected to the following studies.

\section{Morphological study}

Dissections of the nasal cartilages were done as follows:

- a midline skin incision on the dorsum of the nose were done making two flaps;

- the flaps then raised on both sides of the nose from the root to the tip of the nose to expose the subcutaneous tissue;

- the subcutaneous tissue was dissected to expose the nasal cartilages which then dissected then removed.

The exposed cartilages were examined for the shape, parts and attachment.

\section{Morphometric study}

Upon exposure of the nasal cartilages, the upper lateral and lower lateral (alar) cartilages measurements were done. For each cartilage the length, width and thickness was determined. Regarding the lower lateral cartilage the mentioned three parameter measurements were done for its three parts (lateral, middle and medial crura). The measurements were done using simple ruler and Vernier calliper [14] and the greatest length, width and thickness were measured according to Li et al. [21] (Fig. 1):

- ULC length - the distance between upper and lower medial ends of upper lateral cartilages;

- ULC width - the widest distance between medial border and apex of upper lateral cartilage;

- ULC thickness - the greatest thickness of the cartilage;

- lower lateral crus (LLC) length - the distance from distal end of lateral crus to the angle of alar cartilage;

- LLC width - width of lateral crus at the midpoint;

- LLC thickness - the greatest thickness of the cartilage;

- lower medial crus (LMC) length - the distance from ventral end of medial crus to the angle of alar cartilage;

- LMC width - width of medial crus at the midpoint;

- LMC thickness - the greatest thickness of the cartilage;

- middle crus (MC) length - the distance from medial end of lateral crus to the anterior end of medial crus;

- MC width - width of medial crus at the midpoint;

- MC thickness - the greatest thickness of the cartilage; 


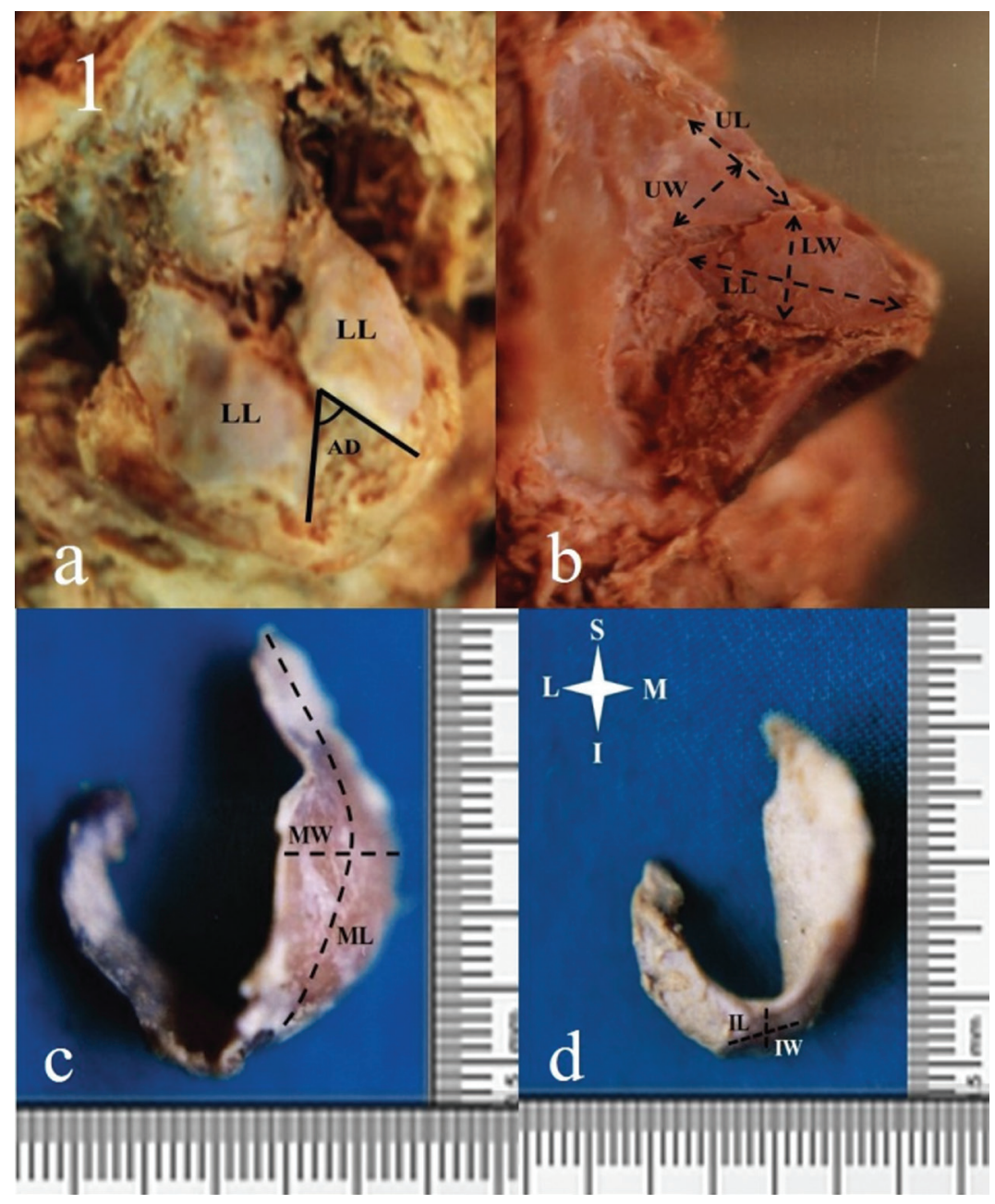

Figure 1. Photographs of a dissected nose with: Panel a: superior view showing the angle of diversion (AD); Panel b: lateral view showing the measurements of upper and lower lateral cartilages where: UL — the length of upper lateral cartilage; UW — the width of upper lateral cartilage; LL — the length of lower lateral crus cartilage; LW — the width of lower lateral crus cartilage; Panel c: lower lateral cartilage showing the measurements of medial crus: ML — lower medial crus length; MW — medial crus width; Panel d: lower lateral cartilage showing the measurements of intermediate (middle) crus: IL — middle crus length; IW — middle crus width.

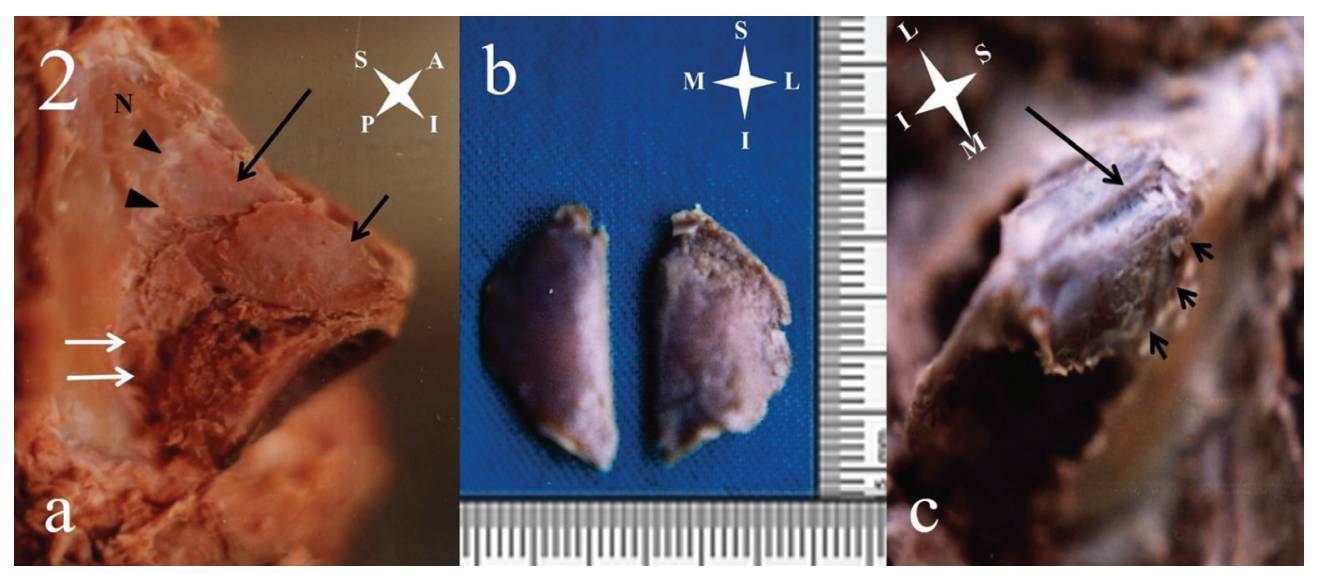

Figure 2. Photographs of a dissected nose (a) and (c), upper lateral nasal cartilage (b) showing: Panel a: triangular, convex upper lateral cartilage (long black arrow) having a base directed medially attached to nasal septum and apex directed laterally attached to lateral margin of piriform fossa (arrow heads). The upper border of the cartilage underlay the nasal bone (N) while the lower border underlay the lower lateral cartilages (long black arrow). Note the small rounded accessory cartilages (white arrows) lying behind the lower lateral cartilage. The lower lateral cartilage (short black arrow) appeared elliptical in shape with long axis directed backward and laterally; Panel b: triangular, convex upper lateral cartilage having a base directed medially and apex directed laterally with lateral surface slightly convex outward; Panel c: triangular, convex upper lateral cartilage having a base directed medially attached to nasal septum (long arrow) and apex directed laterally attached to lateral margin of piriform fossa (short arrows). 
- intercrural angle of divergence (AD) - the angle between the middle parts of alar cartilages of both sides.

\section{Statistical analysis}

The measurements were recorded and statistically analysed with IBM-PC compatible computer using SPSS (version 21) software and student t-test. The p-value less than 0.05 were considered significant.

\section{RESULTS}

\section{Morphological study}

On examination of the cartilaginous skeleton of the dorsum and tip of the nose it was found to be formed of upper lateral cartilage, lower lateral cartilage and small accessory cartilages (Fig. 2a).

Upper lateral cartilage. On examination it appeared triangular in shape with base, apex and two borders

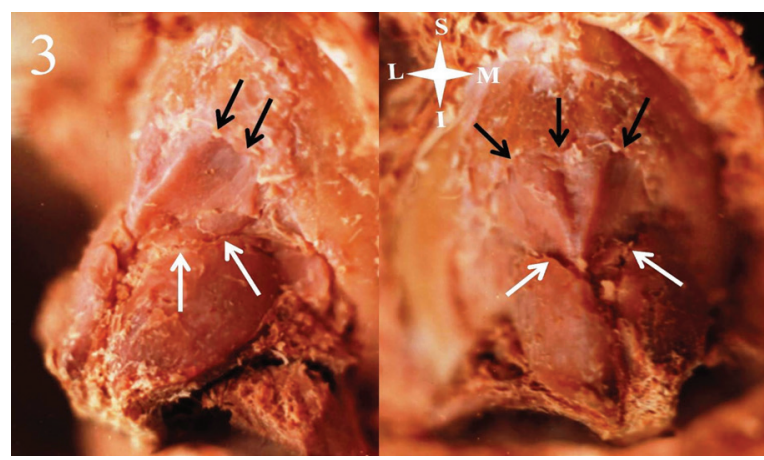

Figure 3. A photograph of superior and side view of a dissected nose showing the convex upper lateral cartilages underlay the nasal bones (black arrows) superiorly and underlay the lower lateral cartilages (white arrows) inferiorly in the scroll area between the upper and lower lateral cartilages. Note the convex lateral crura of the alar cartilage.
(Fig. 2b). The base directed medially attached to nasal septum while the apex directed laterally attached to lateral margin of piriform fossa (Fig. 2c). The upper border of the cartilage underlay the nasal bones while the lower border also underlay the lower lateral cartilages in the scroll area between the upper and lower lateral cartilages (Fig. 3). The ULC was convex in 24 out of 30 (80\%) cases (Figs. 2, 3, 4a), flat in 4 out of $30(13.5 \%)$ (Fig. 4b) and concave in 2 out of 30 (6.5\%) (Fig. 4c).

Lower lateral cartilage. It is formed of three parts: medial, lateral and middle crus. The junction between lateral and middle crus showing angular change in the direction can be named as lateral genu while another one present at the junction between the middle crus and medial crus and also is named as medial genu (Fig. 5a). The lateral crus is elliptical in shape with long axis directed backward and laterally (Figs. 2a, 5b). The lateral crus is leaf-like and larger than the medial one. The medial crus bends posteriorly, then laterally flares posterosuperiorly away from nasal rim to connect with the accessory cartilages (Figs. 5a, 6c). The medial crura held together by ligamentous tissue in columella, oriented in the sagittal plane (Fig. 6a, b). On examination, it was found that in 27 out of 30 (90\%) cases the lateral crura were convex (Figs. 3, 5a, c, 6a), concave in $6.5 \%$ (Fig. $5 \mathrm{~d}$ ) and flat in 3.5\% (Fig. 5b). In 26 out of 30 (87\%) the middle crura were flat (Fig. 5a) and convex in the rest of specimens (13\%) (Fig. 6b, c). The medial crus was convex in 26 out of 30 (87\%) of the cadavers (Fig. $5 \mathrm{a}$ ) but flat in te rest of examined specimens (13\%) (Fig. 6b), with no significant gender difference. The space between the lower lateral cartilages (interdomal space) is filled with fat forming the

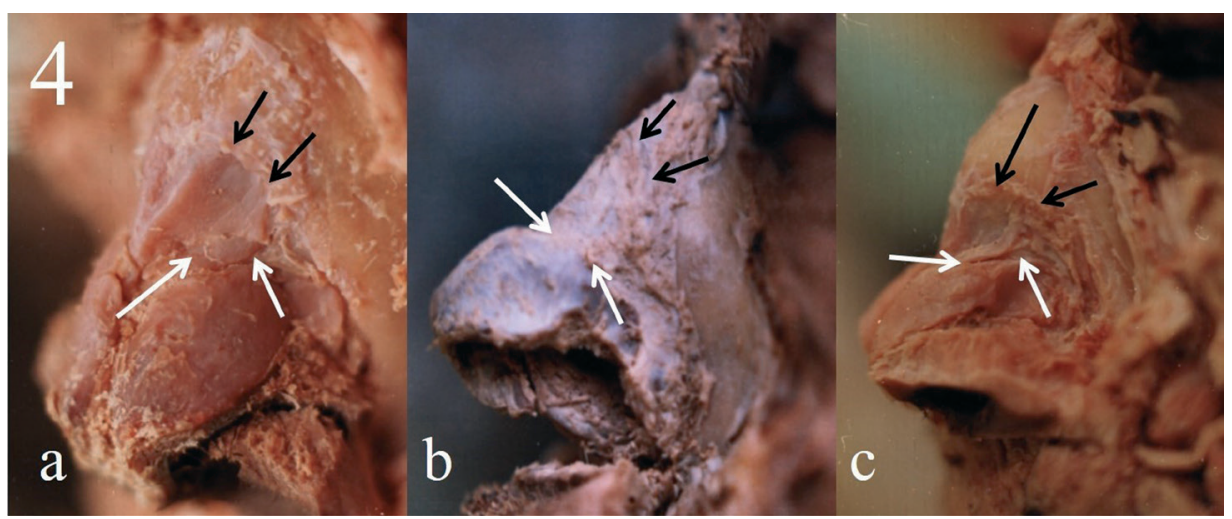

Figure 4. A photograph of superior and side view of a dissected nose showing: Panel a: a convex upper lateral cartilages underlay the nasal bones (black arrows) superiorly and underlay the lower lateral cartilages (white arrows) inferiorly in the scroll area between the upper and lower lateral cartilages; Panel b: a flat upper lateral cartilages underlay the nasal bones (black arrows) superiorly and underlay the lower lateral cartilages (white arrows) inferiorly in the scroll area between the upper and lower lateral cartilages; Panel c: a concave upper lateral cartilages underlay the nasal bones (black arrows) superiorly and underlay the lower lateral cartilages (white arrows) inferiorly in the scroll area between the upper and lower lateral cartilages. 


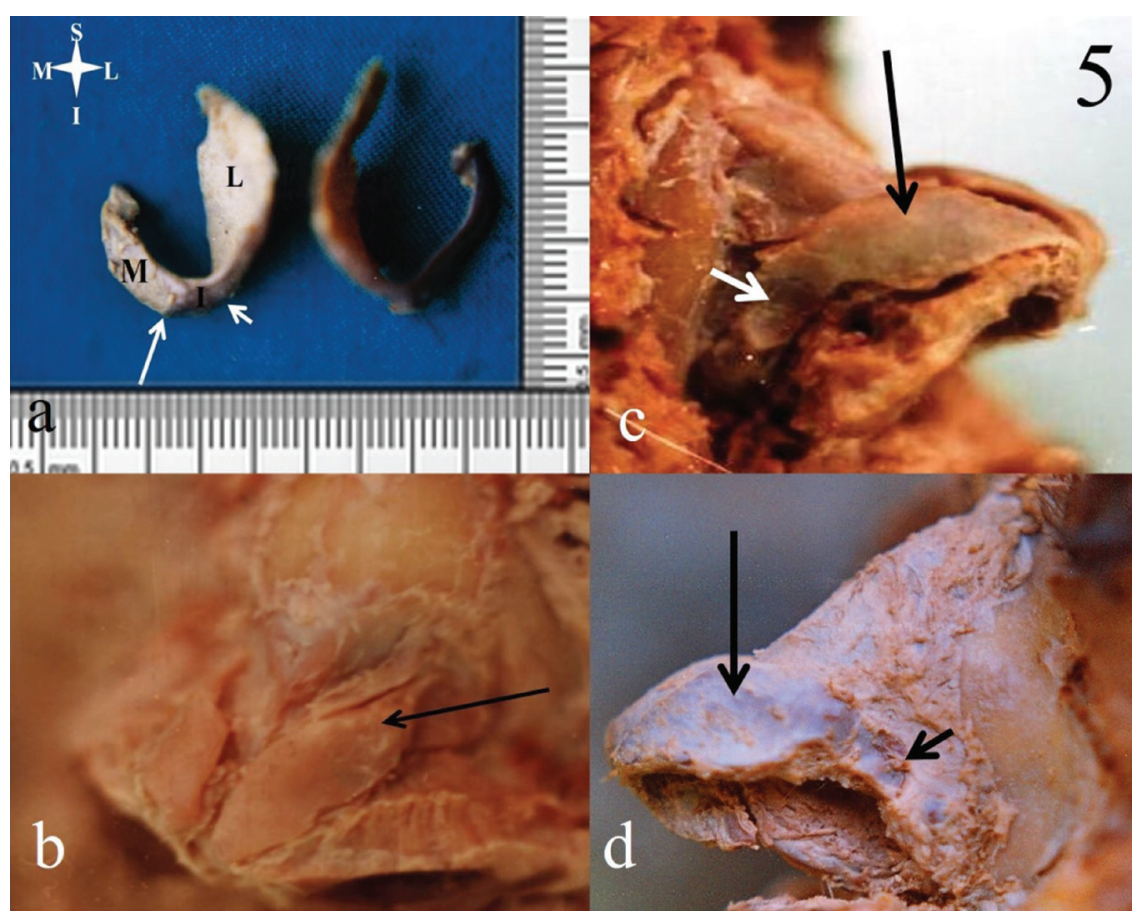

Figure 5. Photographs of a lower lateral nasal cartilage (a), superior view (b) and side view (c, d) of dissected nose showing: Panel a: the three parts forming the cartilage; medial (M), lateral (L) and intermediate (I) crura. The junction between lateral and intermediate crus showing angular change in the direction can be named as lateral genu (short arrow) while another one present at the junction between the intermediate and medial crus and also is named as medial genu (long arrow). The lateral crus is leaf-like and larger than the medial one. The medial crus bends posteriorly then laterally flare posterosuperiorly away from nasal rim to connect with the accessory cartilages. Note the convex lateral crura of the alar cartilage; Panel b: the lateral crus (arrow) is elliptical in shape with long axis directed backward, upward and laterally. Note the flat lateral crura of the alar cartilage; Panels c, $\mathbf{d}$ : the lateral crus (long arrow) is elliptical in shape with small extension that project posterosuperior (short arrow). Note the convex lateral, flat middle and convex medial crura of the alar cartilage in panel a and concave in panel $\mathrm{d}$.

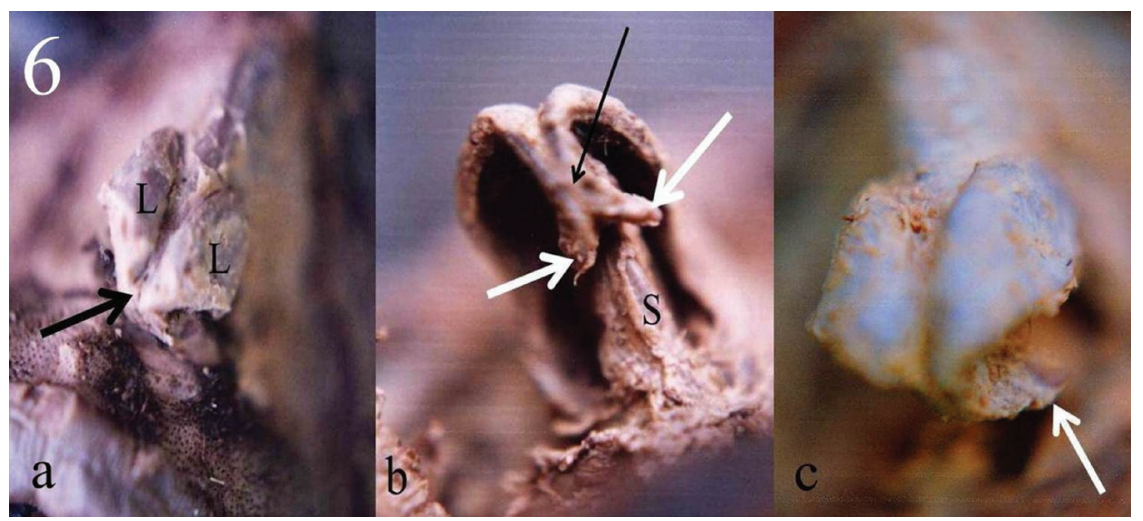

Figure 6. Photographs of a superior view $(\mathbf{a}, \mathbf{c})$ and inferior view (b) of a dissected nose showing. Panel a: the medial crura of the lower lateral cartilages (L) of both sides held together by ligamentous tissue (arrow) in columella, oriented in the sagittal plane. Note the convex lateral crura of the alar cartilage; Panel b: the medial crura of the lower lateral cartilages of both sides held together by ligamentous tissue (black arrow) in columella, oriented in the sagittal plane. The medial crus (white arrow) bends posteriorly then laterally flare posterosuperiorly away from nasal rim. Note the nasal septum (S), the convex middle and flat medial cura; Panel c: the medial crus (white arrow) bends posteriorly then laterally flare posterosuperiorly away from nasal rim. Note the convex middle crus.

dome of the tip of the nose. A pad fat was present in most of cases - 28 out of 30 (93.5\%) (Fig. 7a) and apparently absent in the rest of cases (6.5\%) (Fig. 7b, c). The amount of fat was variable.
Accessory cartilages. Appeared as small rounded cartilages (Fig. 2a) lying behind the lower lateral cartilage between lateral crus and pyriform aperture and found nearly in all examined specimens. 


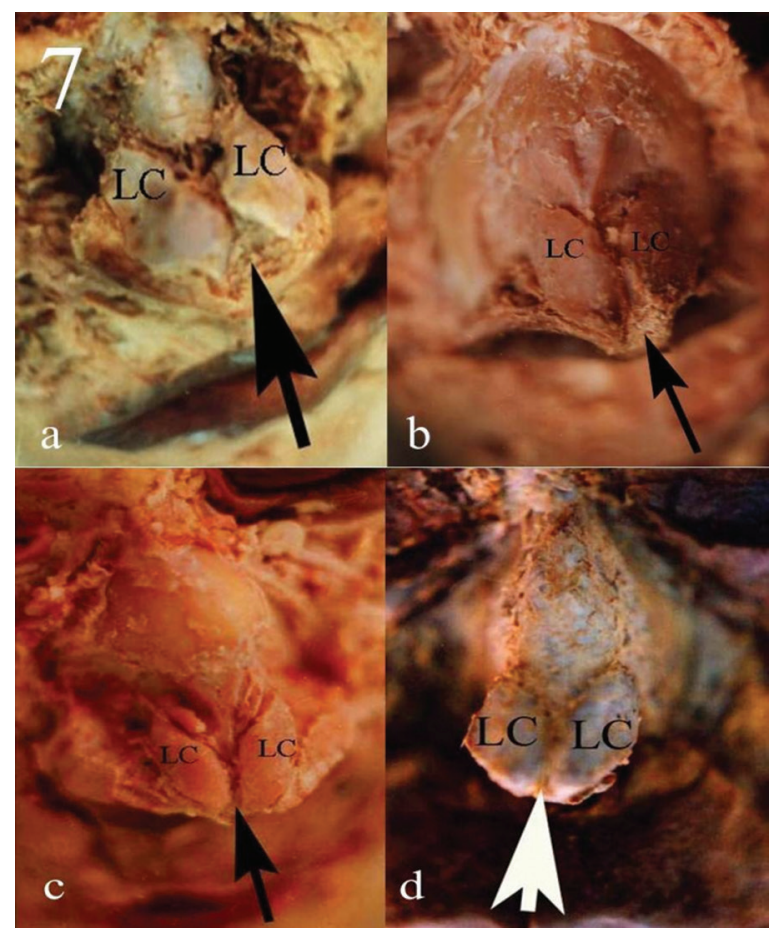

Figure 7. Photographs of superior view of a dissected nose showing: Panels a, b: presence of interdomal fat (arrow) filling the space between the two lower lateral nasal cartilages (LC); Panels c, d: apparently absence of interdomal fat (arrow) in the space between the lower lateral cartilages of both sides (LC).

\section{Morphometric study}

Upper lateral cartilage measurements. The measurements of the upper lateral nasal cartilages were summarised in Table 1. It revealed that the total range of length of the right ULC was $15.8-27.5 \mathrm{~mm}$ while that of the left was 15.2-29 $\mathrm{mm}$ with total mean $22.6 \pm 1.5$ and $21.11 \pm 1.93$, respectively. The range of length of the right ULC under the nasal bone was $2.9-7 \mathrm{~mm}$ while that of the left side was $2.4-$ $-6.6 \mathrm{~mm}$ with total mean $2.87 \pm 1.34$ and $3.0 \pm 1.06$, respectively (Table 1 ). The total range of width of the right ULC was 10-15 mm while that of the left was 9-15 mm with total mean $12.8 \pm 1.29$ and $12.72 \pm$ \pm 1.6 , respectively. The total range of thickness of the right ULC was $0.4-1.6 \mathrm{~mm}$ while that of the left was $0.4-1.5 \mathrm{~mm}$ with total mean $1.34 \pm 0.14$ and $1.42 \pm$ \pm 0.15 , respectively.

Lower lateral cartilage measurements. The measurements of the lower lateral nasal cartilages were summarised in Table 2 . It revealed that the total range of length of the right lower lateral cartilage lateral crus was 17-25 mm while that of the left was 16-25 mm with total mean $22.4 \pm 2.1$ and $22.76 \pm$ \pm 2.5 , respectively. The total range of length of the right lower lateral cartilage $M C$ was $5-9 \mathrm{~mm}$ while that of the left was $4-10 \mathrm{~mm}$ with total mean $7.06 \pm$ \pm 1.14 and $7.25 \pm 1.09$, respectively. The total range of length of the right lower lateral cartilage medial crus was 4-10 $\mathrm{mm}$ while that of the left was 4-9 $\mathrm{mm}$ with total mean $6.4 \pm 0.86$ and $6.46 \pm 0.90$, respectively.

The total range of width of the right lower lateral cartilage lateral crus was $8-13 \mathrm{~mm}$ while that of the left was 8-14 $\mathrm{mm}$ with total mean $10.6 \pm 1.29$ and $11.72 \pm 1.3$, respectively. The total range of width of the right lower lateral cartilage MC was 3-6 $\mathrm{mm}$ while that of the left was $3-6 \mathrm{~mm}$ with

Table 1. The mean, total mean and range of length, width and thickness of the upper lateral cartilages in both sexes in $\mathrm{mm}$

\begin{tabular}{|c|c|c|c|c|}
\hline & \multicolumn{4}{|c|}{ Upper lateral cartilage } \\
\hline & \multicolumn{2}{|c|}{ Total } & \multicolumn{2}{|c|}{ Under nasal bone } \\
\hline & Right & Left & Right & Left \\
\hline \multicolumn{5}{|c|}{ Total length: upper lateral cartilage length } \\
\hline Male & $22.23 \pm 1.26$ & $20.63 \pm 2.66$ & $3.41 \pm 1.38$ & $3.62 \pm 1.12$ \\
\hline Female & $23.10 \pm 1.73$ & $21.6 \pm 1.21$ & $2.16 \pm 1.30$ & $2.45 \pm 1.0$ \\
\hline Total mean & $22.6 \pm 1.5$ & $21.11 \pm 1.93$ & $2.87 \pm 1.34$ & $3.0 \pm 1.06$ \\
\hline Total range & $15.8-27.5$ & $15.2-29$ & $2.9-7$ & $2.4-6.6$ \\
\hline \multicolumn{5}{|c|}{ Width: upper lateral cartilage width } \\
\hline Male & $13.16 \pm 1.98$ & $13.30 \pm 2.01$ & & \\
\hline Female & $12.44 \pm 1.16$ & $12.23 \pm 1.28$ & & \\
\hline Total mean & $12.8 \pm 1.29$ & $12.72 \pm 1.6$ & & \\
\hline Total range & 10-15 & $9-15$ & & \\
\hline \multicolumn{5}{|c|}{ Thickness: upper lateral cartilage thickness } \\
\hline Male & $1.76 \pm 0.14$ & $1.54 \pm 0.18$ & & \\
\hline Female & $0.93 \pm 0.10$ & $0.94 \pm 0.32$ & & \\
\hline Total mean & $1.34 \pm 0.14$ & $1.42 \pm 0.15$ & & \\
\hline Total range & $0.4-1.6$ & 0.4-1.5 & & \\
\hline
\end{tabular}


Table 2. The mean \pm standard deviation of length, width and thickness (in $\mathrm{mm}$ ) of the lower lateral (alar) cartilages in both sexes

\begin{tabular}{|c|c|c|c|c|c|c|}
\hline & \multicolumn{2}{|c|}{ Lateral crus } & \multicolumn{2}{|c|}{ Middle crus } & \multicolumn{2}{|c|}{ Medial crus } \\
\hline & Right & Left & Right & Left & Right & Left \\
\hline \multicolumn{7}{|l|}{ Length } \\
\hline Male & $21.75 \pm 2.1$ & $21.62 \pm 1.4$ & $7.23 \pm 1.15$ & $7.32 \pm 1.1$ & $6.5 \pm 0.95$ & $6.5 \pm 0.93$ \\
\hline Female & $23.10 \pm 1.73$ & $23.9 \pm 2.07$ & $6.9 \pm 1.14$ & $7.20 \pm 1.09$ & $6.31 \pm 0.80$ & $6.43 \pm 0.93$ \\
\hline Total mean & $22.4 \pm 2.1$ & $22.76 \pm 2.5$ & $7.06 \pm 1.14$ & $7.25 \pm 1.09$ & $6.4 \pm 0.86$ & $6.46 \pm 0.90$ \\
\hline Total range & $17-25$ & $16-25$ & 5-9 & 4-10 & 4-10 & 4-9 \\
\hline \multicolumn{7}{|l|}{ Width } \\
\hline Male & $11.10 \pm 1.42$ & $12.2 \pm 1.38$ & $4.5 \pm 0.67$ & $4.5 \pm 0.66$ & $3.55 \pm 0.92$ & $3.52 \pm 0.97$ \\
\hline Female & $10.11 \pm 0.53$ & $11.25 \pm 0.75$ & $4.0 \pm 0.69$ & $4.07 \pm 0.67$ & $2.91 \pm 0.33$ & $2.90 \pm 0.87$ \\
\hline Total mean & $10.6 \pm 1.29$ & $11.72 \pm 1.3$ & $4.25 \pm 0.60$ & $4.2 \pm 0.7$ & $3.23 \pm 0.71$ & $3.21 \pm 0.90$ \\
\hline Total range & 8-13 & 8-14 & 3-6 & 3-6 & 2-5 & 3-5 \\
\hline \multicolumn{7}{|l|}{ Thickness } \\
\hline Male & $0.9 \pm 0.11$ & $0.95 \pm 0.16$ & $0.9 \pm 0.85$ & $0.8 \pm 0.92$ & $1.0 \pm 0.1$ & $1.0 \pm 0.07$ \\
\hline Female & $0.8 \pm 0.12$ & $0.9 \pm 0.15$ & $0.85 \pm 0.12$ & $0.9 \pm 0.15$ & $0.80 \pm 0.17$ & $0.93 \pm 0.14$ \\
\hline Total mean & $0.8 \pm 0.14$ & $0.92 \pm 0.15$ & $0.87 \pm 0.13$ & $0.85 \pm 0.13$ & $0.9 \pm 0.12$ & $0.96 \pm 0.11$ \\
\hline Total range & $0.4-1.4$ & $0.5-1.5$ & $0.4-1.0$ & $0.4-1.2$ & $0.5-1.6$ & $0.5-1.4$ \\
\hline
\end{tabular}

Table 3. The mean \pm standard deviation and range of the intercrural angle of divergence of the lower lateral (alar) cartilages in both sexes

\begin{tabular}{lcccc}
\hline & Male & Female & Mean & Range \\
\hline Angle of divergence & $40.21 \pm 3.82$ & $38.3 \pm 2.60$ & $39.2 \pm 3.9$ & $23-44$ \\
\hline
\end{tabular}

total mean $4.25 \pm 0.60$ and $4.2 \pm 0.7$, respectively. The total range of width of the right lower lateral cartilage medial crus was $2-5 \mathrm{~mm}$ while that of the left was 3-5 mm with total mean $3.23 \pm 0.71$ and $3.21 \pm 0.90$, respectively.

The total range of thickness of the right lower lateral cartilage lateral crus was $0.4-1.4 \mathrm{~mm}$ while that of the left was $0.5-1.5 \mathrm{~mm}$ with total mean $0.8 \pm 0.14$ and $0.92 \pm 0.15$, respectively. The total range of thickness of the right lower lateral cartilage MC was $0.4-1.0 \mathrm{~mm}$ while that of the left was $0.4-1.2 \mathrm{~mm}$ with total mean $0.87 \pm 0.13$ and $0.85 \pm 0.13$, respectively. The total range of thickness of the right lower lateral cartilage medial crus was $0.5-1.6 \mathrm{~mm}$ while that of the left was $0.5-1.4 \mathrm{~mm}$ with total mean $0.9 \pm 0.12$ and $0.96 \pm 0.11$, respectively. Statistically there was no significant differences between genders and sides regarding length, width and thickness of upper and lower lateral cartilages.

Intercrural angle of divergence. In the present study in measuring the AD between the middle parts of alar cartilages of both sides ranged $23-44^{\circ}$ with the mean $39.2 \pm 3.9^{\circ}$ being $40.21 \pm 3.82^{\circ}$ in male and $38.3 \pm 2.60^{\circ}$ in female (Table 3 ).

\section{DISCUSSION}

Due to the complexity of the nose and its role in facial recognition it is an important feature for both forensic and surgical craniofacial reconstruction [3]. However, Frederick [16] stated that that the nasal tip is a three dimensional structure of curves and angles. The underlying alar cartilages support and configure the tip. The shape, size and position of the paired alar cartilages are reflected through the skin as surface anatomy.

Upon examination of the Egyptian nasal cartilages, in the current study, morphologically revealed that the ULC was triangular in shape with base, apex and two borders. The base directed medially attached to nasal septum while the apex directed laterally attached to lateral margin of piriform fossa. The upper border of the cartilage underlay the nasal bones while the lower border also underlay the lower lateral cartilages in the scroll area between the upper and lower lateral cartilages. The ULC was convex in 24 out of 30 (80\%) cases, flat in 4 out of $30(13.5 \%)$ and concave in 2 out of 30 (6.5\%).

In the current study the measurements of the upper lateral nasal cartilage revealed that the total 
range of length of the right ULC was $15.8-27.5 \mathrm{~mm}$ while that of the left was $15.2-29 \mathrm{~mm}$ with total mean $22.6 \pm 1.5$ and $21.11 \pm 1.93$, respectively. The range of length of the right ULC under the nasal bone was $2.9-7 \mathrm{~mm}$ while that of the left side was 2.4-6.6 mm with total mean $2.87 \pm 1.34$ and $3.0 \pm$ \pm 1.06 , respectively. This finding is markedly less and disagreed with those of Oneal et al. [22] who stressed on the fact that the ULC extends for 7-10 mm under the nasal bones.

The total range of width of the right ULC was 10-15 mm while that of the left was $9-15 \mathrm{~mm}$ with total mean $12.8 \pm 1.29$ and $12.72 \pm 1.6$, respectively. The total range of thickness of the right ULC was $0.4-1.6 \mathrm{~mm}$ while that of the left was $0.4-1.5 \mathrm{~mm}$ with total mean $1.34 \pm 0.14$ and $1.42 \pm 0.15$, respectively.

de Pochat et al. [13] in their study on 16 specimens (13 male and 3 female) observed that length of the ULC portion under the nasal bones on the right side ranged from $3 \mathrm{~mm}$ to $7 \mathrm{~mm}(4.62 \pm 1.20 \mathrm{~mm})$. On the left side it ranged from $2 \mathrm{~mm}$ to $7 \mathrm{~mm}(4.56 \pm$ $\pm 1.26 \mathrm{~mm}$ ). The total length of the upper lateral cartilages ranged from $16 \mathrm{~mm}$ to $28 \mathrm{~mm} \mathrm{(20.44 \pm}$ $\pm 3.26 \mathrm{~mm}$ ) on the right side and $17 \mathrm{~mm}$ to $30 \mathrm{~mm}$ $(20.75 \pm 3.71 \mathrm{~mm})$ on the left side.

The examination of lower lateral (alar) cartilage showed that it is formed of three parts: medial, lateral and middle crus. The junction between lateral and middle crus showing angular change in the direction can be named as lateral genu while another one present at the junction between the middle crus and medial crus and also is named as medial genu. The lateral crus is elliptical in shape with long axis directed backward and laterally. The lateral crus is leaf-like and larger than the medial one. The medial crus bends posteriorly then laterally flares posterosuperiorly away from nasal rim to connect with the accessory cartilages. The medial crura held together by ligamentous tissue in columella, oriented in the sagittal plane. On examination it was found that in 27 out of $30(90 \%)$ cases the lateral crura were convex and in 26 out of $30(87 \%)$ the middle crura were flat. The medial crus was convex in 26 out of $30(87 \%)$ cadavers with no significant gender difference.

According to the present study, the lateral crus is convex in approximately $90 \%$ of the Egyptians. It is nearly the same morphology of the Caucasians $[2,9]$ and Iranians (87\%) [15]. It is convex in 33\% of Afri- can Americans [26] and concave in $40 \%$ of Korean women and $50 \%$ of Korean men [20]. Consequently, the lateral crura of the Egyptian population (both male and female) are nearly similar to the Persian one but differ from those of other races. In the current work the average length of the lower lateral cartilage lateral crus was $22.4-22.7 \mathrm{~mm}$ and the average width was 10.6-11.7 $\mathrm{mm}$. The average length, width and thickness were nearly similar in Egyptian male and female cadavers. These measurements less than the Persian in length (23-24 mm) but approximated in width $(10.5-11 \mathrm{~mm})$. The present measurements approximated the average width reported by Snyder [26] for African American subjects (12 mm) 16-18 mm; however, the average length was shorter in African Americans $(18 \mathrm{~mm})$ than in Egyptian. The average width in Egyptian cadavers $(11.1 \mathrm{~mm})$ was higher to the width found in Koreans $(10.2 \mathrm{~mm})$, but the length (22.4-22.7 mm) and thickness $(0.8-0.9 \mathrm{~mm})$ in Egyptian were greater than the length (16.8$-19.7 \mathrm{~mm})$ and thickness $(0.54-0.55 \mathrm{~mm})$ in Koreans. Also, the thickness of lateral crura in men was nearly similar that in women, which was same as the Korean study done by Dhong and Han [12] and Kim et al. [19] but differed from Farahvash et al. [15] work who reported that the thickness was bigger in male than females.

The shape of the MC in the present work was flat in the majority of specimen (87\%). This finding agreed with those of Farahvash et al. [15] who observed that the middle crura were flat in $80 \%$ to $90 \%$ of cadavers. Studies from other countries have not examined the shape of the MC. In the current work the medial crus was convex bilaterally in 26 out of 30 (87\%) Egyptian cadavers, with no significant gender difference. That was different in other ethnic groups being convex in $58 \%$ in Persian [15], nearly 50\% in Koreans [12] in Americans there was three morphological types the commonest are concavoconvex and convex (83\%) [9] . Daniel [10] added that the cartilage was symmetrical in $50 \%$ of specimens.

Morphometrically in the current work, the total range of length of the right lower lateral cartilage medial crus was 4-10 $\mathrm{mm}$ while that of the left was 4-9 mm with total mean $6.4 \pm 0.86$ and $6.46 \pm 0.90$, respectively. This value is smaller than those observed by Dhong and Han [12] in Asian group where the length was $8.7 \pm 1.37$ but nearly similar to Persian population $(6.8 \pm 0.99)$ according to Farahvash et 
al. [15]. The total range of width of the right lower lateral cartilage medial crus in Egyptian was $2-5 \mathrm{~mm}$ while that of the left was 3-5 $\mathrm{mm}$ with total mean $3.23 \pm 0.71$ and $3.21 \pm 0.90$, respectively. The value was also smaller Asian ( $4.3 \pm 0.69)$ and Persian (3.45 \pm 0.92 ) groups. The total range of thickness of the right lower lateral cartilage medial crus in Egyptian was $0.5-1.6 \mathrm{~mm}$ while that of the left was $0.5-1.4$ $\mathrm{mm}$ with total mean $0.9 \pm 0.12$ and $0.96 \pm 0.11$, respectively. Different values were recorded in different ethnic groups, being $0.7 \mathrm{~mm}$ in Caucasians [18], $0.5 \mathrm{~mm}$ in Asian [12] and $0.9 \mathrm{~mm}$ in Persian [15]. The finding nearly similar to those of Persian.

On the other hand, there was no significant statistical differences between genders regarding upper and lower lateral cartilages. This in contrary differed from those of Farahvash et al. [15] and Dhong and Han [12] who found significant differences between sexes in measurements of the alar cartilage.

The present study showed that the space between the lower lateral cartilages (interdomal space) is filled with fat forming the dome of the tip of the nose. This fat was variable in amount and present in most of cases $(93.5 \%)$ and apparently absent in the rest of (6.5\%). Coskun et al. [6] disagreed with that as they demonstrated that all their studied cases had a fat pad in the interdomal space with varying sizes. More than that Copcu et al. [5] discussed that all patients had a fat pad in the interdomal space, of varying sizes, but fatty (bulbous) noses had larger fat pads. The size of the fat pads varied from $1.2 \mathrm{~mm} \times 2.4 \mathrm{~mm}$ to $3.6 \mathrm{~mm} \times 5.2 \mathrm{~mm}$. They speculate that the interdomal fat pad is an important anatomical structure and may contribute to unexpected postoperative results in rhinoplasty.

The accessory cartilages in the present work appeared as small rounded cartilages lying behind the lower lateral cartilage between lateral crus and piriform aperture and found nearly in all examined specimens. On the other hand, Farahvash et al. [15] found small amounts of accessory cartilage at the end of the lateral crus in $89 \%$ of the cadavers. Similarly, accessory cartilages have been reported among Korean patients [25]. In Caucasians, accessory cartilages at the end of the LLC were found to continue to the piriform aperture [17].

In the present study in measuring the AD between the middle parts of alar cartilages of both sides ranged between $23^{\circ}$ and $44^{\circ}$ with the mean $39.2^{\circ} \pm$ \pm 3.9 being $40.21^{\circ} \pm 3.82$ in males and $38.3^{\circ} \pm 2.60$ in females. The angle is less than that described by
Toriumi [27] being $45^{\circ}$. This angle to a great extent can partially predict together with domal fat the shape of the tip of the nose [8].

Comprehensive understanding of nasal anatomy and the relationships among its individual structures are essential for performing a successful rhinoplasty. Patients must see a visible cosmetic improvement, without compromised function. Furthermore, both the cosmetic and functional result must be durable. This is a truly challenging part of rhinoplasty but it can be accomplished with a solid understanding of the nasal anatomy and relationships of the different anatomical components.

\section{CONCLUSIONS}

It can be concluded from the current study that Egyptian nasal cartilages were different morphologically and morphometrically from those in Asians, Caucasians, Persian and African Americans. The differences among ethnic groups should be put into consideration and it is recommended when operating on the nasal tip and dorsum of the nose, in order to reduce the possibility of secondary corrective procedures.

\section{Limitations of the study}

The main limitation of the present work is that the number of the cadavers used could not exceed 30 .

\section{Acknowledgements}

It is my great honour to express my deepest gratitude and grateful thanks to our cadavers who still teach us.

\section{REFERENCES}

1. Adamson PA (1993) Nasal tip surgery in open rhinoplasty. Facial Plastic Surgery Clinics of North America, 1: 39-52.

2. Ali-Salaam P, Kashgarian M, Davila J, Persing J (2002) Anatomy of the Caucasian alar groove. Plast Reconstr Surg, 110: 261-271.

3. Anderson KJ, Henneberg M, Norris RM (2008) Anatomy of the nasal profile. J Anat, 213: 210-216.

4. Chin KY, Uppal R (2014) Improved access in endonasal rhinoplasty: the cross cartilaginous approach. J Plast Reconstr Aesthet Surg, 67: 781-788.

5. Copcu E, Metin K, Özsunar Y, Çulhaci N, Özkök S (2004) The interdomal fat pad of the nose: a new anatomical structure. Surg Radiol Anat, 26: 14-18.

6. Coskun N, Yavuz AD, Alparslan DM, Sindel T, Islamoglu K, Sindel M (2008) Three-dimensional measurements of the nasal interdomal fat pad. Aesthetic Plastic Surg, 32: 262-265.

7. Crag JR, Bied A, Landas S, Suryadevara A (2015) Anatomy of the upper lateral cartilage along the lateral piriform aperture. Plast Reconstr Surg, 135: 406-411. 
8. Daniel RK (1992) The nasal tip: anatomy and aesthetics. Plastic Reconstructive Surg, 89: 216-224.

9. Daniel RK (2009) Middle Eastern rhinoplasty in the United States: Part I. Primary rhinoplasty. Plast Reconstr Surg, 124: $1630-1639$.

10. Daniel RK (2009) Middle Eastern rhinoplasty in the United States: Part II. Primary rhinoplasty. Plast Reconstr Surg, 124: $1640-1648$.

11. Daniel RK, Letourneau A (1988) Rhinoplasty: nasal anatomy. Ann Plast Surg, 20: 5-13.

12. Dhong S, Han SK (2002) Anthropometric study of alar cartilage in Asian. Ann Plast Surg, 48: 386-391.

13. de Pochat VD, Alonso N, Ribeiro EB, da Rocha EA, Tenorio EA, Meneses JV (2012) Anatomical variations of the upper lateral cartilage and their implications in rhinoplasty. Aesthetic Plast Surg, 36: 285-289.

14. Dion MC, Jafek BW, Tobin CE (1978) The anatomy of the nose: external support. Arch Otolaryngol, 104: 145-150.

15. Farahvash MR, Ebrahimi A, Farahvash B, Farahavash $Y$ (2012) Anatomic and anthropometric analysis of 72 lower lateral nasal cartilages from fresh Persian (Iranian) cadavers. Aesthet Surg J, 32: 447-453.

16. Frederick JM (1999) Anatomic reconstruction of the nasal tip cartilages in secondary and reconstructive rhinoplasty. Plastic Reconstructive Surg, 104: 2187-2198.
17. Guyuron B, Behmand RA (2003) Nasal tip sutures. Plast Reconstr Surg, 112: 1130-1145.

18. Hatzis GP, Sherry SD, Hogan GM, Finn RA (2004) Observations of the marginal incision and lateral crura alar cartilage asymmetry in rhinoplasty: a fixed cadaver study. Oral Surg Oral Med Oral Pathol Oral Radiol Endod, 97: 432-437.

19. Kim CH, Jung DH Park, MN, Yoon JH (2010) Surgical anatomy of cartilaginous structures of the Asian nose: clinical implications in rhinoplasty. Laryngoscope, 120: 914-919.

20. Kyung K (2006) Factors affecting nostril shape in Asian nose. Plastic Reconstr Surg, 7: 118.

21. Li AQ, Sun YG, Wang GH, Zhong ZK (2002) Anatomy of the nasal cartilages of the unilateral complete cleft lip nose. Plast Reconstr Surg, 109: 1835-1838.

22. Oneal RM, Beil RJ, Schlesinger J (1999) Surgical anatomy of the nose. Otolaryngol Clin North Am, 32: 1.

23. Rohrich RJ, Ghavami A (2009) Rhinoplasty for Middle Eastern noses. Plast Reconstr Surg, 123: 1343-1354.

24. Rowe-Jones J (2014) Refining the nasal tip: an anatomical approach. Facial Plast Surg, 30: 113-122.

25. Seung K, Dong G (2004) An anatomic study of nasal tip supporting structures. Ann Plast Surg, 52: 134-139.

26. Snyder GB (1971) Rhinoplasty in the Negro. Plast Reconstr Surg, 47: 572.

27. Toriumi DM (2006) New concepts in nasal tip contouring. Arch Facial Plast Surg, 8: 156-185. 